\title{
QUALITY FACTOR SENSITIVITY TO CRYSTALLOGRAPHIC AXIS MISALIGNMENT IN SILICON MICROMECHANICAL RESONATORS
}

\author{
A. K. Samarao and F. Ayazi
}

Georgia Institute of Technology, Atlanta, Georgia, USA

\begin{abstract}
We study the sensitivity of quality factor in single crystal silicon (SCS) micromechanical resonators to crystallographic axis misalignments that are present due to fabrication non-idealities. Our experimental results, being reported for the first time here, unveil that very small angular misalignment from [110] axis of transduction adversely affects the high Q of a bulk acoustic wave SCS resonator by more than $50 \%$, unlike the misalignment errors about the [100] axis of transduction. Interestingly, when the axis of transduction is intentionally offset by a large angle from either [100] or [110], multiple peaks with comparable relative strength are observed from a single resonator.
\end{abstract}

\section{INTRODUCTION}

Single crystal silicon (SCS) micromechanical resonators have shown high frequencies $(>100 \mathrm{MHz})$ and high quality factors $(Q>50,000)$, surpassing those of commercially available quartz resonators [1]. Insertion of such high frequency silicon resonators into low phase-noise frequency references requires stable and reproducible $Q$. However, the observed $Q$ variation across various batches of simultaneously processed devices under very similar processing conditions suggests that there might be a possible influence from a non-systematic non-ideality in the fabrication process that might be adversely affecting the $Q$. While many studies have been conducted on determining the various energy loss mechanisms limiting the $Q$ of a SCS resonator [2], the sensitivity of $Q$ to crystallographic misalignment has not yet been systematically studied.

(a)
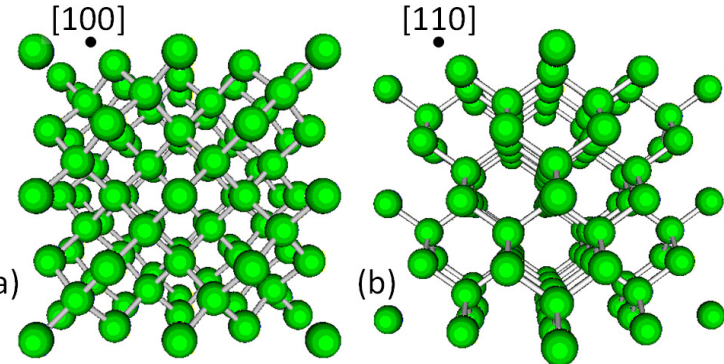

Fig. 1: Schematic showing the arrangement of atoms in the silicon crystal lattice as seen along the (a) [100] and (b) [110] directions.

Every atom in silicon has a linear chain of atoms aligned along the [100] or [110] direction which facilitates efficient transmission of the compressional and dilational forces of an acoustic wave (Figure 1). Hence, SCS microresonators are typically transduced along either of these axes. However, any angular misalignment from these two axes of transduction disrupts the atomic linearity which might lead to acoustic losses at the atomic level, the ensemble of which might reflect on the $Q$ of the resonator. As will be revealed from this work, the amount of angular misalignment needed to adversely affect the $Q$ of the resonator is imperceptible to the human eye and are beyond the tolerance limits of automated lithography systems as well.

A rectangular silicon bulk acoustic resonator (or SiBAR) (Figure 2(a)) that involves a capacitive air-gap based transduction along a very specific crystallographic axis in SCS is the best candidate to study variations in $Q$ with very small angular offsets from the intended axes of transduction. The SiBAR is placed between the drive and sense electrodes separated by a very high aspect-ratio air-gap realized using the HARPSS process [3]. A DC polarization voltage $\left(V_{p}\right)$ applied to the resonator generates an electrostatic field in these capacitive gaps. When an AC voltage is applied to the drive electrode, the resulting time-varying electrostatic force applied to the corresponding face of the resonator induces an acoustic wave that propagates through the bar, resulting in a width-extensional resonance mode (Figure 2(b)) whose frequency is primarily defined by the width of the SiBAR. Small changes in the air gap on the other side of the device induce a voltage on the sense electrode whose amplitude peaks at the mechanical resonance frequency.

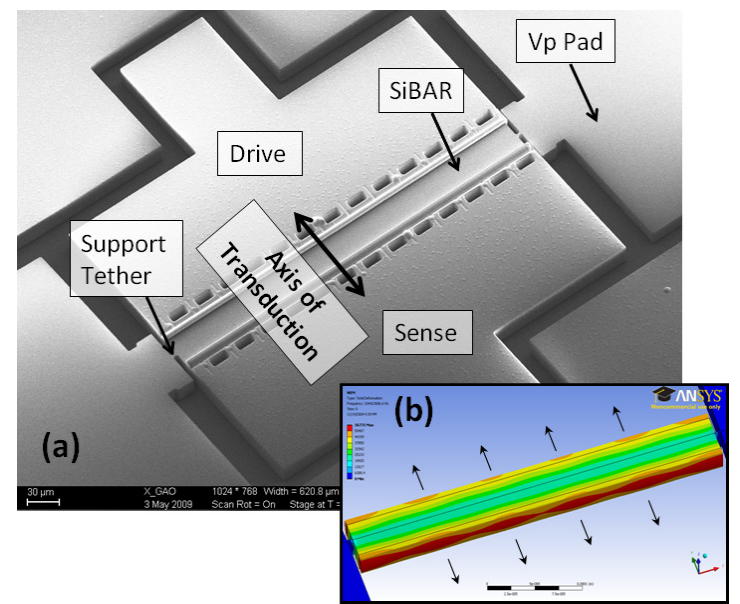

Fig. 2: (a) SEM and (b) Simulated width-extensional mode (WEM) shape of the SiBAR.

Experimental platform to study $\boldsymbol{Q}$ sensitivity to angular offset

A batch of $100 \mathrm{MHz}$ SiBARs (width $=40 \mu \mathrm{m}$, thickness $=20$ $\mu \mathrm{m}$ and length $=10 \times$ width) were fabricated about both the [110] and [100] axes of transduction with intentionally created negative and positive angular offsets in steps of 0.1 degrees as illustrated in Figure 3.

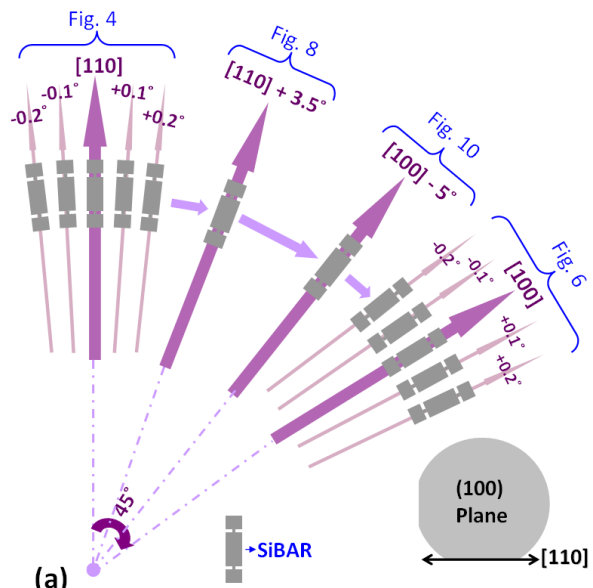

Solid-State Sensors, Actuators, and Microsystems Workshop Hilton Head Island, South Carolina, June 6-10, 2010 


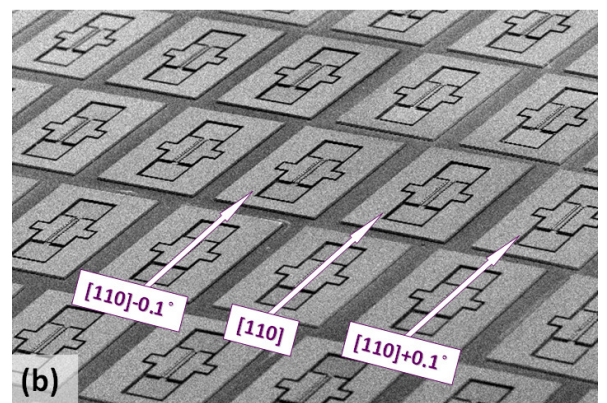

Fig. 3: (a) Schematic and (b) SEM of the intentionally angular offset $100 \mathrm{MHz}$ SiBARs for studying the $Q$ variation with angular misalignment.

The variation in $Q$ with the doping level of the SCS substrate and the size of support tethers needs to be taken into account to attribute any variation in $Q$ solely to the angular misalignment. Hence, these SiBARs were fabricated across various batches of wafers that spanned different levels of boron doping (0.01 0.02 $\Omega$-cm [moderately- doped], 0.001 0.002 $\Omega$-cm [highlydoped] $\&<0.001 \Omega$-cm [ultra-highly-doped]) as purchased from the vendor for different widths of the support tethers $(3 \mu \mathrm{m} \& 1.5$ $\mu \mathrm{m})$. Any pattern in $Q$ variation observed across all these batches of devices can be safely concluded to stem from the angular misalignment in the axis of transduction.

\section{$Q$ VARIATION ABOUT [110] AXIS OF TRANSDUCTION}

Among the SiBARs fabricated about the [110] axis, the SiBAR that offers the highest $Q$ is assumed to be transduced exactly along [110]. This assumption is reasonable as the atomic arrangement favoring minimum loss for acoustic transduction would exist only for the [110] axis of transduction. The SiBARs that are angularly offset clockwise from the [110] device are assumed to have a positive offset and vice versa (Figure 3 ). A very interesting symmetric pattern in the $Q$ variation with angular offsets has been observed in these devices about [110] as shown in Figure 4 . These results correspond to moderately doped substrate with a supporting tether width of $3 \mu \mathrm{m}$. The $Q$ values were measured in vacuum at an input power of $-10 \mathrm{dBm}$ at a $V_{p}$ of $10 \mathrm{~V}$, and a capacitive air-gap of $\sim 100 \mathrm{~nm}$. Except for a minor variation in each $Q$ value by $\pm 3 \mathrm{k}$, this exact pattern repeats in highly doped and ultra-highly-doped substrates as well, thus confirming the pattern to be a result of angular misalignment.

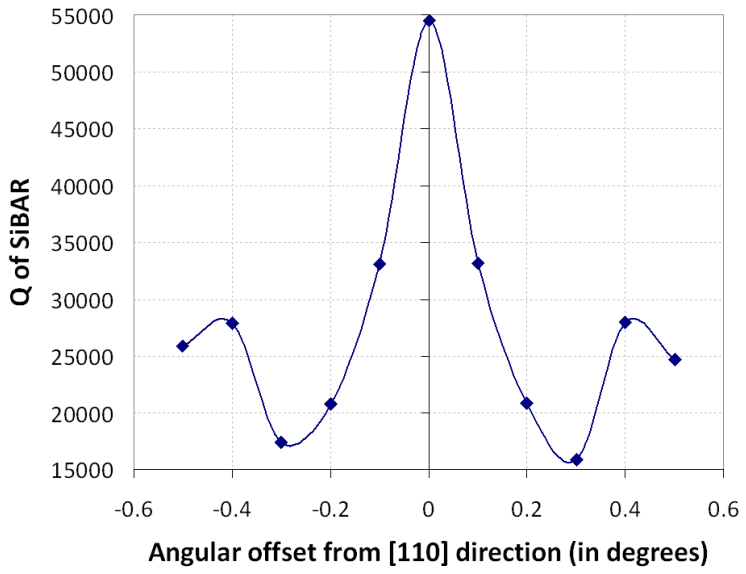

Fig. 4: Measured pattern of $Q$ variation in SiBARs with angular offsets from [110] axis of transduction.
The most important conclusion from this study is that the $Q$ drops by $\sim 50 \%$ when offset from the [110] axis of transduction by just 0.1 degree. This calls for additional efforts to alleviate misalignment sensitivity when $Q$ is of utmost importance. A $180^{\circ}$ phase difference has been measured across all the resonance peaks to confirm that the lower- $Q$ is not due to a coupling of adjacent resonant modes of the SiBAR. When these devices were fabricated with a $1.5 \mu \mathrm{m}$ wide supporting tether to further reduce the support loss, the unloaded $Q$ (i.e., $Q$ at the turn-ON $V_{p}$ of $1 \mathrm{~V}$ ) along the [110] direction almost matches the maximum possible $f Q$ in SCS (Figure 5(a)) [4]. Figure 5(b) illustrates that other than the expected $\sim 50 \%$ drop with a 0.1 degree angular offset, spurious modes start appearing within a span of $1 \mathrm{MHz}$ from the main resonance peak. One or more of such spurs have been observed in all the devices about the [110] axis except for the one aligned exactly long [110]. Thus, the observation of such spurs can serve to be a good indicator of the existence of an angular misalignment from the fabrication process. A vacuum measurement setup and careful SOLT calibration have been found to be necessary in the case of some devices to observe the spurs.

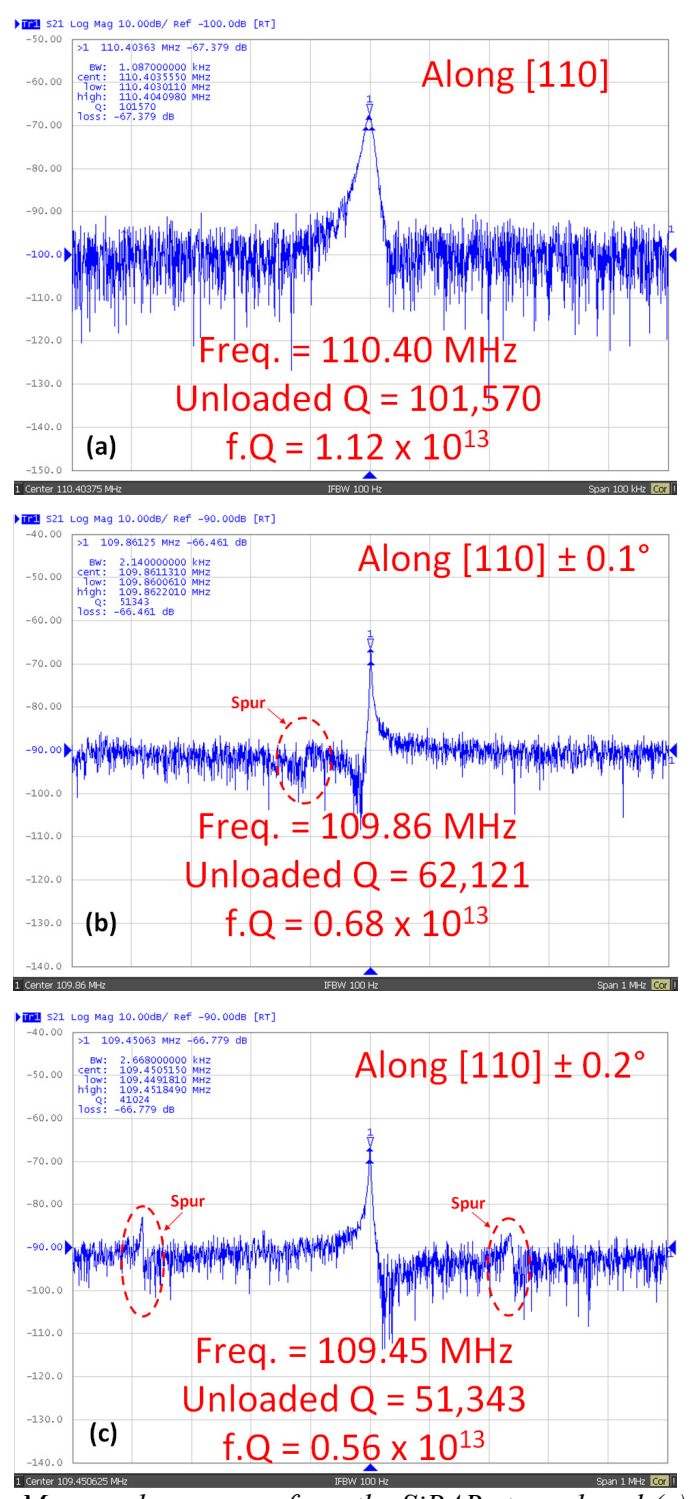

Fig. 5: Measured responses from the SiBARs transduced (a) along [110] direction, and along $(b) \pm 0.1^{\circ}$ and $(b) \pm 0.2^{\circ}$ angular offset. 


\section{$Q$ VARIATION ABOUT [100] AXIS OF TRANSDUCTION}

Unlike [110], the SiBARs about [100] were not observed to show any pattern in $Q$ variation with angular offsets from the [100] axis of transduction (Figure 6). The data in Figure 6 corresponds to the devices about [100] but are from the same wafer and under the same measurement conditions as that of Figure 4. A random variation about $10 \%$ in the $Q$ value was observed in these devices making [100] axis of transduction the best option when repeatability of $Q$ across various batches of fabrication is of prime importance. Such a random Q variation of $\sim 10 \%$ in the devices about [100] was observed across the highly-doped and ultra-highly doped substrates as well. The absence of a pattern in $Q$ variation about [100] unlike [110] can be attributed to the comparatively high atomic density along the (100) plane over the (110) plane (Figure 1). As a result, the disruption of atomic periodicity with angular offsets in the case of [110] axis of transduction is more drastic compared to [100], which might be leading to sharper patterns in $Q$ variations.

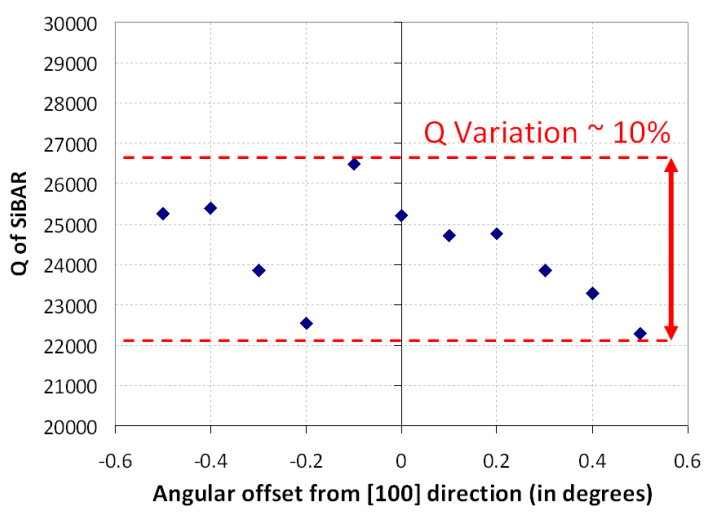

Fig. 6: Measured random $Q$ variation of $\sim 10 \%$ in SiBARs with angular offsets from [100] axis of transduction.

Figure 7 shows a typical $\mathrm{SiBAR}$ fabricated with a $1.5 \mu \mathrm{m}$ wide supporting tether (same wafer as Figure 5) and transduced about [100] at a $V_{p}$ of $10 \mathrm{~V}$. The unloaded $Q$ for such devices randomly varies between $60 \mathrm{k} \sim 70 \mathrm{k}$. Spurs are observed in a span of $5 \mathrm{MHz}$ in all the devices about [100] except for the one aligned exactly along [100]. In both the devices about [110] and [100], the spurs become more prominent with increasing $V_{p}$ similar to the main resonance peak. . The $Q$ value observed exactly along [100] axis of transduction $(\sim 65 \mathrm{k})$ is smaller in comparison to [110] ( $\sim 101 \mathrm{k})$ which is in agreement with the Akheiser regime of the silicon $f Q$ product [4].

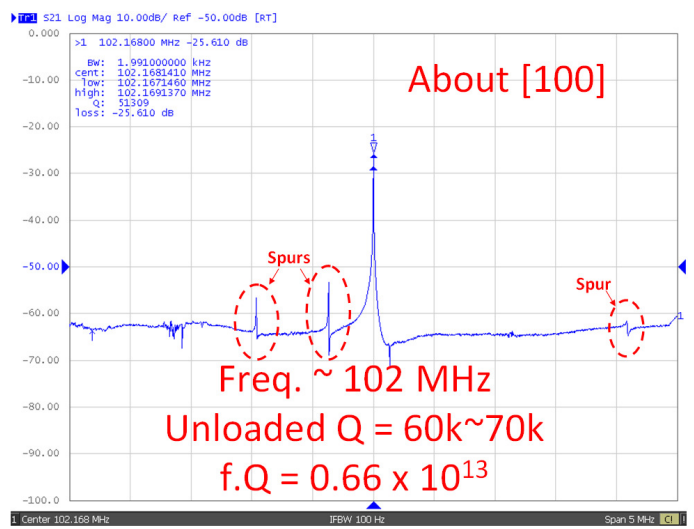

Fig. 7: Typical measured response from the SiBARs transduced about [100] axis of transduction.

\section{SPURIOUS MODES BETWEEN [110] AND [100] AXES OF TRANSDUCTION}

The geometry of the SiBAR and the configuration of its drive/sense electrodes do not favor the excitation of out-of-plane or torsional modes of resonance. Hence, the spurious resonance modes also need to be predominantly confined to those similar to the primary width-extensional mode (WEM) (Figure 2(b)). However, the effective width as seen by the acoustic wave may differ, albeit slightly, depending on the chain of atoms favored for propagation. As a result, they give rise to spurious widthextension-like resonance modes very near the main resonance peak when transduced at an angular offset from [110] or [100]. However, the acoustic impedance for these spurious modes are typically very large compared to the primary WEM along [110] and [100] which usually makes them very weak in comparison (i.e., larger insertion loss). Interestingly, there exist certain axes of transduction between [110] and [100] where two or more such $W E M$-like resonance modes can be equally or comparably favored, giving rise to multiple strong peaks from a single SiBAR.

For example, at an angular offset of $+3.5^{\circ}$ from [110] axis of transduction (Figure 8), four comparable peaks can be seen in a span of $1 \mathrm{MHz}$ at a $V_{p}$ of $10 \mathrm{~V}$, each varying considerably in $Q$ and insertion loss $(I L)$. No regular patterns have been observed in the relative strength or spectral spacing or number of these multiple peaks with increasing angular offsets from [110] or [100]. The temperature coefficient of frequency $(T C F)$ of the multiple peaks are found to vary only within $\pm 2 \mathrm{ppm} /{ }^{\circ} \mathrm{C}$ across all such measured angular offset devices and correspond to the range of TCF expected from the respective doping level of the substrates [5]. However, the same multiple peak responses are repeatable across batches of fabrication varying in doping levels and tether widths.

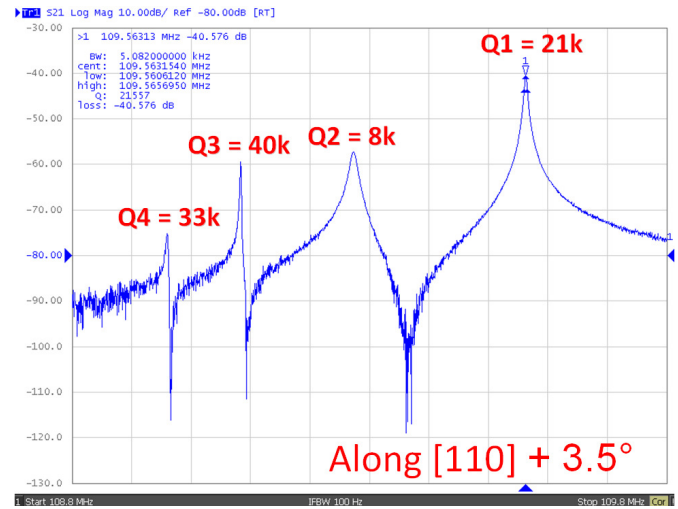

Fig. 8: Measured response from a SiBAR transduced along [110] $+3.5^{\circ}$ axis of transduction.

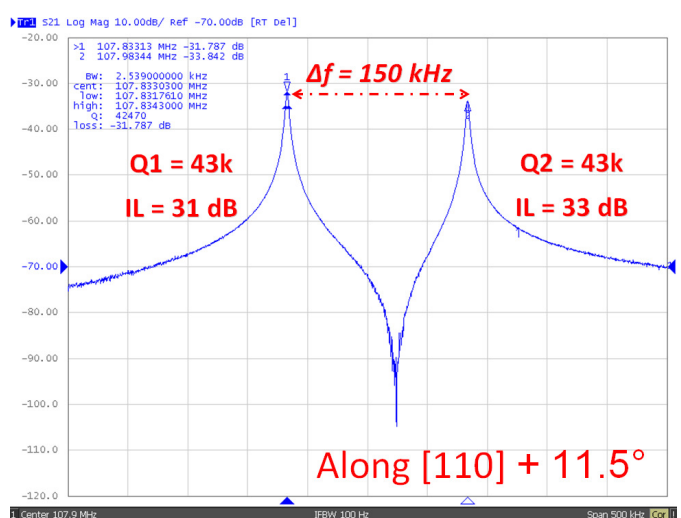

Fig. 9: Measured response from a SiBAR transduced along [110] $+11.5^{\circ}$ axis of transduction. 
Two equally strong peaks (IL $\sim 31 \mathrm{~dB}$ ) separated by $150 \mathrm{kHz}$ with the same $\mathrm{Q}$ of $43 \mathrm{k}$ has been observed from a single SiBAR transduced at an offset of $+11.5^{\circ}$ from [110] axis of transduction and at a $V_{p}$ of $10 \mathrm{~V}$ (Figure 9). It is worth noting that achieving such small frequency separations with the same $Q$ from an array of two micromechanical resonators is challenging otherwise due to the tolerance limits of microfabrication processes [6].

Another interesting observation from SiBARs that are $5^{\circ}$ and $5.1^{\circ}$ offset respectively from the [100] axis of transduction is shown in Figure 10. Transduction along $5^{\circ}$ offset from [100] shows two very prominent, equally strong peaks $(I L \sim 30 \mathrm{~dB})$ at a $V_{p}$ of $10 \mathrm{~V}$ separated by $486 \mathrm{kHz}$ as shown in Figure 10(a), among other minor spurs. One of the peaks exhibits a $Q$ of $74 \mathrm{k}$ which is more than twice that of the other strong peak with a $Q$ of $35 \mathrm{k}$. Interestingly, the SiBAR that is transduced at $5.1^{\circ}$ offset from [100] has only one strong peak at the same $V_{p}$ of $10 \mathrm{~V}$ as shown in Figure 10(b) among other minor spurs. The $Q$ of this single strong peak is an average of the $Q$ s of the two peaks in the $5^{\circ}$ offset device, and its $I L$ of $24 \mathrm{~dB}$ is $6 \mathrm{~dB}$ better. Also, the resonance frequency at $5.1^{\circ}$ offset approximates to be in the middle of the two peaks from the $5^{\circ}$ offset device. These reflect that a transitional acoustic path of transduction might be favored in the $5.1^{\circ}$ offset device wherein the larger $Q$ from the $5^{\circ}$ offset device has decreased while the smaller $Q$ has increased. However, such a transitional acoustic path seems to half the motional impedance, leading to twice a voltage gain at the sense electrode which reflects as a $6 \mathrm{~dB}$ improvement in $I L$. Such observations hold the key to understanding the transduction along non-conventional axes which might hold possible to a new type of coupling at the atomic level that offers low-loss SCS electromechanical resonators and resonator-array functionalities within a very small footprint.

\section{DISCUSSIONS}

The study of $Q$ variation with angular misalignment can have a high risk of error due to the non-idealities of the silicon wafer manufacturing processes, even prior to the fabrication of the SiBARs. The tolerances involved in the thickness and planarity of the sliced wafers from the silicon ingot and the subsequent manufacturing of the SOI layers introduce unpredictability in precisely determining the axis of transduction in these SiBARs. Hence, all reported trends in $Q$ variation in this work have been cross-checked across multiple fabrication batches. The systematic variations from the DRIE and photolithography processes and their effects on the dimensions of the resonator and the support tether have been minimized to the extent that trends in $Q$ variations reported from adjacent devices with $\leq \pm 0.5^{\circ}$ angular offset become independent of process tolerances. Other dissipative mechanisms that limit the $Q$ (i.e., thermo-elastic damping, support loss, etc.) are assumed to be identical for the SiBARs positioned across minor angular offsets.

\section{CONCLUSION}

Three major conclusions can be drawn from this work. First, among the SCS resonators transduced about the [110] direction, the maximum achievable $Q$ is observed only while transduced exactly along the [110] direction. A $50 \%$ reduction in $Q$ occurs even when the axis of transduction is angularly offset by $0.1^{\circ}$, which calls for additional efforts to alleviate misalignment sensitivity when $Q$ is of utmost importance. Second, no such observable pattern has been observed in resonators transduced about the [100] direction. The variation in $Q$ is random and is smaller than $10 \%$, suggesting [100] direction to be a better choice of transduction axis when $Q$ reproducibility across various batches of fabrication is desirable. Finally, two or more dominant peaks have been observed from a single SiBAR when offset by a relatively larger angle from either [110] or [100] axis of transduction. It may be possible to choose a particular axis of transduction that might offer a new type of electromechanical coupling at the atomic level for the realization of low-loss resonators and resonator-array type of functionalities.

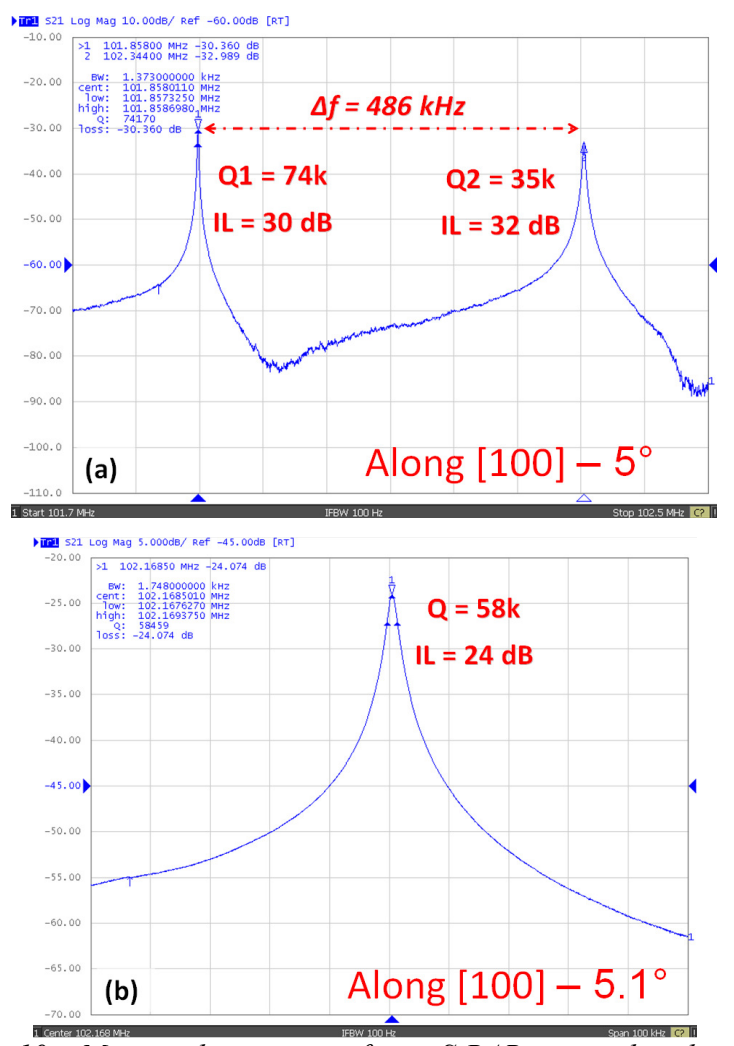

Fig. 10: Measured response from SiBARs transduced along (a) $[100]-5^{\circ}$ and (b) $[100]-5.1^{\circ}$ axis of transduction.

\section{ACKNOWLEDGEMENTS}

This work was supported by Integrated Device Technology (IDT).

\section{REFERENCES}

[1] F. Ayazi, "MEMS for Integrated Timing and Spectral Processing," Invited Paper, Proc. IEEE Custom Integrated Circuits Conference (CICC 2009), pp. 65-72.

[2] R. N. Candler, H. Li, M. Lutz, W. T-. Park, A. Partridge, G. Yama, and T. W. Kenny, "Investigation of Energy Loss Mechanisms in Micromechanical Resonators," Transducers 2003, pp. 332-335.

[3] S. Pourkamali, G. K. Ho, and F. Ayazi, "Low-Impedance VHF and UHF Capacitive Silicon Bulk Acoustic Wave Resonators - Part I: Concept and Fabrication," IEEE Transactions on Electron Devices, vol. 54, no. 8, Aug. 2007, pp. 2017-2023.

[4] R. Tabrizian, M. Rais-Zadeh, and F. Ayazi, "Effect of Phonon Interactions on Limiting the $f Q$ Product of Micromechanical Resonators," Transducers 2009, pp. 2131-2134.

[5] A. K. Samarao and F. Ayazi, "Temperature Compensation of Silicon Micromechanical Resonators via Degenerate Doping," IEEE International Electron Devices Meeting (IEDM 2009), pp. 789-792.

[6] A. K. Samarao and F. Ayazi, "Post Fabrication Electrical Trimming of Silicon Bulk Acoustic Resonators using Joule Heating," IEEE International Conference on Micro Electro Mechanical Systems (MEMS 2009), pp. 892-895.

\section{CONTACT}

A. K. Samarao, tel: +1-404-385-3291; ashwins@ece.gatech.edu 